\title{
All-Optical Radio Frequency Spectrum Analyzer Based on Cross-Phase Modulation in a Silicon-Rich Nitride Waveguide
}

\author{
M. Rezagholipour Dizaji ${ }^{1}$, C. J. Krückel ${ }^{2}$, P. A. Andrekson ${ }^{2}$, V. Torres-Company ${ }^{2}$, and L. R. Chen ${ }^{1}$ \\ ${ }^{1}$ Department of Electrical and Computer Engineering, McGill University, Montreal, Quebec, Canada, H3A0E9 \\ ${ }^{2}$ Department of Microtechnology and Nanoscience (MC2), Chalmers University of Technology, 41296 Gothenburg, Sweden \\ Mohammad.rezagholipourdizaji@mail.mcgill.ca
}

\begin{abstract}
We report RF-spectrum analysis of optical signals based on cross-phase-modulation in a silicon-rich nitride waveguide. Measurements show a bandwidth of at least $560 \mathrm{GHz}$ for our RF-spectrum analyzer. RF-spectra measurements for pulse trains at rates from $\sim 10 \mathrm{GHz}$ to $\sim 160 \mathrm{GHz}$ are demonstrated.
\end{abstract}

Keywords - Radio frequency spectrum analysis, cross phase modulation, integrated optics, silicon nitride.

\section{INTRODUCTION}

The increasing demand for high speed data processing requires devices and techniques that are not limited by the speed of electronics. One of the microwave photonic applications that highlights the potential of nonlinear optical effects to overcome the bandwidth limitation of electronics is the monitoring of the radio frequency (RF) of an optical signal. Traditionally, the RF spectrum of an optical signal is measured by using a combination of a high bandwidth photodetector and an electrical RF spectrum analyzer (RFSA). However, the bandwidth of signals that can be characterized using this approach is limited by the available bandwidth from the photodetectors and/or RFSA, unless some form of mixing technique (down-conversion) is used. Optical techniques can overcome this bandwidth limitation and include intensity autocorrelation and the use of cross-phase modulation (XPM) to implement a photonic RFSA [1]. In the latter, owing to the nonlinear interaction between the signal under test and a continuous wave $(\mathrm{CW})$ probe, the phase of the $\mathrm{CW}$ probe will be modulated by the intensity of the signal under test. This results in XPM-based spectral broadening about the $\mathrm{CW}$ probe's wavelength. This newly generated optical spectrum centered at the $\mathrm{CW}$ probe, uniquely represents the RF spectrum of the signal under test and can be recorded with an optical spectrum analyzer. The bandwidth of this technique is limited by the nonlinear effect and the nonlinear medium, and the resolution is limited to the resolution of the optical spectrum analyzer (OSA) that is used to record the XPM spectrum.

XPM-based RFSA can be performed in a variety of nonlinear media, however integrated optical signal processing and microwave photonics by reducing the overall size and easier control of dispersion compared to nonlinear fibres have been of particular interest during the past years. Integrated photonic RFSA was first demonstrated using chalcogenide waveguides [2] and shortly after using silicon nanowires [3]. In [4] photonic RFSA with higher bandwidth using CMOS

This research is supported in part by the Natural Sciences and Engineering Research Council of Canada, the Swedish Research Council and STINT (Swedish Foundation for International Cooperation in Research and Higher Education) compatible platforms (Hydex) is reported. Implementation of microwave photonic modules in silicon nitride has also attracted many attentions in the past decade $[5,6]$ but photonic RFSA has not been implemented in this platform yet.

$\mathrm{Si}_{3} \mathrm{~N}_{4}$ has a wide transparency window from UV to shortwavelength infrared and therefore can operate over an ultrabroad wavelength range. For example, $\mathrm{Si}_{3} \mathrm{~N}_{4}$ is more suitable for applications at $850 \mathrm{~nm}$ compared to SOI as the latter has high absorption at wavelengths below $1100 \mathrm{~nm}$. While $\mathrm{Si}_{3} \mathrm{~N}_{4}$ has a lower nonlinear refractive index compared to chalcogenide [7] and SOI [8], the possibility of reducing the propagation loss (a loss as low as $0.1 \mathrm{~dB} / \mathrm{m}$ in thin waveguides was demonstrated in [9]) and the capability of handling high power levels makes it an attractive platform. Most importantly, in contrast with $\mathrm{SOI}, \mathrm{Si}_{3} \mathrm{~N}_{4}$ is not expected to have nonlinear loss and two-photon absorption (TPA) in the telecommunication range due to its larger bandgap energy [10]. This is valid for Hydex as well [4].

Proper dispersion engineering of $\mathrm{Si}_{3} \mathrm{~N}_{4}$ by varying the geometry of the waveguide will be needed to optimize nonlinear effects such as self-phase modulation, XPM, and four wave mixing (FWM) [11]. For rectangular waveguides in order to obtain proper phase matching condition over the desired wavelength range of operation, a thickness of more than $\sim 600 \mathrm{~nm}$ is required. Increasing the thickness of the waveguide will increase the chance of cracking during film deposition. We resolved this problem, by increasing the relative content of silicon and changing the stoichiometry of the $\mathrm{Si}_{\mathrm{x}} \mathrm{N}_{\mathrm{y}}$ film during the deposition process. Using this process we obtained a crack-free silicon-rich nitride (SRN) waveguides with a thickness $>700 \mathrm{~nm}$ using a single deposition step. Our SRN waveguides have a nonlinear coefficient that is 5 times greater than that for stoichiometric silicon nitride $\left(\mathrm{Si}_{3} \mathrm{~N}_{4}\right)$ as well as the possibility to achieve anomalous dispersion [12]. In [13] we used this SRN waveguides to perform all-optical wavelength conversion based on XPM, and recently demonstrated 1.5 octave supercontinuum generation using femtosecond pulses directly obtained from a fiber oscillator [14].

Our silicon rich nitride platform offers the opportunity of performing nonlinear optics, and implementation of microwave processing applications such as XPM-based RFSA. Indeed, further optimization of the waveguide's design needs to be done for any particular signal processing and microwave photonics application. In the case of photonic RFSA, the 
absence of nonlinear absorption in silicon rich-nitride material systems (similar to Hydex [4]) makes this platform a suitable candidate as the frequency response of the photonic RFSA will not be dependent to the power level of the input signal. Also the broadband operation range of silicon nitride gives us this freedom to be able to obtain XPM that is used for RF spectrum analysis, over a very broader wavelength range. However, proper dispersion engineering for the required phase matching is needed. We report the first operation of an XPM-based RFSA using an integrated silicon nitride waveguide. Measurements of electrical spectra for optical pulse trains with repetition rates from $\sim 10 \mathrm{GHz}$ to $\sim 160 \mathrm{GHz}$ are demonstrated. The measurements show a bandwidth of greater than $560 \mathrm{GHz}$ for our photonic RFSA.

\section{EXPERIMENTS AND RESULTS}

Our SRN waveguide has dimensions $0.7 \mu \mathrm{m} \times 1.65 \mu \mathrm{m}$, with a length of $2 \mathrm{~cm}$. The increased thickness of $700 \mathrm{~nm}$ without cracking was achieved by enriching the silicon content to $65 \%$ (and $35 \%$ of nitrogen) in the $\mathrm{Si}_{\mathrm{x}} \mathrm{N}_{\mathrm{y}}$ film. The waveguide is buried in silicon dioxide and the contrast in the refractive index results in a high confinement of the optical field inside the SRN core with an effective area $\mathrm{A}_{\text {eff }} \sim 0.9 \mu \mathrm{m}^{2}$. The nonlinear coefficient $\gamma$ at $1550 \mathrm{~nm}$ is 4.5 (W.km) ${ }^{-1}$. The waveguide supports both quasi-TE and quasi-TM modes and the overall fiber-to-fiber loss for the quasi-TE mode is $11 \mathrm{~dB}$ which gives an estimated propagation loss of $\sim 1 \mathrm{~dB} / \mathrm{cm}$ (given a coupling loss of $\sim 4.5 \mathrm{~dB} /$ facet [12]) using edge coupling into and out of the waveguide with lensed fibers of $2.5 \mu \mathrm{m}$ spot diameter. The waveguide has a relatively flat and anomalous dispersion over the entire $\mathrm{C}$ - and L-bands: the measured dispersion is $67 \mathrm{ps} /(\mathrm{nm} \cdot \mathrm{km})$ with a dispersion slope of 0.015 $\mathrm{ps} /\left(\mathrm{nm}^{2} \cdot \mathrm{km}\right)$ at $1550 \mathrm{~nm}[13]$.

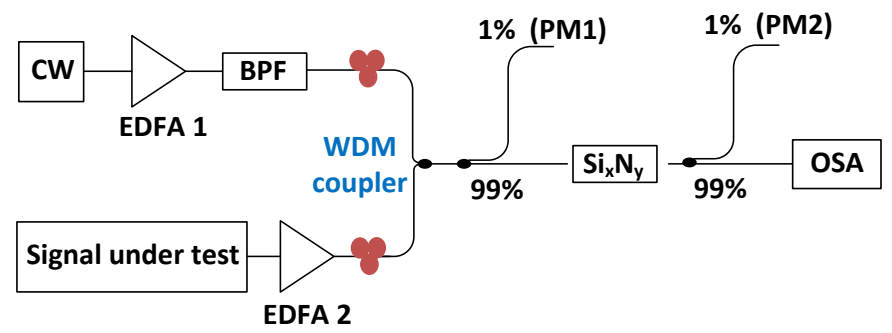

Fig. 1. Experimental setup for the all-optical RF spectrum analyzer, CW: continuous wave, BPF: band pass filter, OSA: optical spectrum analyzer.

The XPM-based RF spectrum analysis technique has an intrinsic bandwidth which depends on the Kerr effect's response time. Other than that, the dispersion profile of the nonlinear medium in which XPM occurs affects the bandwidth of the RF-spectrum analyzer. More specifically, the group velocity mismatch between the $\mathrm{CW}$ probe and the signal under test causes a walk off which limits the operation bandwidth. Considering all these limitations, the operation bandwidth of the photonic RFSA is still far beyond the speed of the state of the art electronics. In order to quantify the bandwidth limitation of our photonic RFSA, we use the method proposed by [1] which is based on mixing two beating $\mathrm{CW}$ signals with a frequency separation $\Delta f$ (around a center frequency $f_{\mathrm{s}}$ ) that interact with a $\mathrm{CW}$ probe centered at the frequency $f_{\mathrm{p}}$. The beating waves form a modulated envelope centered at the CW probe frequency with a detuning of $\Delta f$ on both sides. By varying $\Delta f$ and measuring the corresponding power amplitude of the tones at $f_{\mathrm{p}} \pm \Delta f$ we can determine the operation bandwidth.

The experimental setup of the photonic RFSA is shown in Fig. 1. The same setup is used to estimate the bandwidth of the photonic RFSA, such that in this case the 'signal under test' comprises two tunable laser sources (TLS), with a frequency offset with respect to each other which are then combined together using a 50/50 coupler. The combined signal is amplified using a high power EDFA (EDFA 2) that can provide a maximum output power of $33 \mathrm{dBm}$. The $\mathrm{CW}$ probe signal is generated using a DFB laser source at $1538.9 \mathrm{~nm}$ which is amplified to $18 \mathrm{dBm}$ followed by a $0.4 \mathrm{~nm}$ band pass filter (BPF) to remove the out of band amplified spontaneous emission (ASE) noise. The signals on the two branches are then combined using a WDM coupler with a transmission band of $5 \mathrm{~nm}$ centered at $1550 \mathrm{~nm}$. The signal generated from the two TLS lasers passes through the transmission port of the WDM coupler. The combined signal is then launched into the SRN waveguide. The power of the signal launched into the waveguide, as well as the output signal power from the waveguide are monitored using two power meters. The optical spectra of the output of the waveguide for different frequency detuning $\Delta f$ were captured using an OSA. Frequency detuning $\Delta f$ is varied by symmetrically varying the frequency of the two TLS lasers around $f_{\mathrm{s}}$ which results in varying the frequency of the XPM-RF tones $\left(f_{\mathrm{p}} \pm \Delta f\right)$. The optical spectrum and the peak power of the XPM-RF tones were recorded over a broad range of detuning.

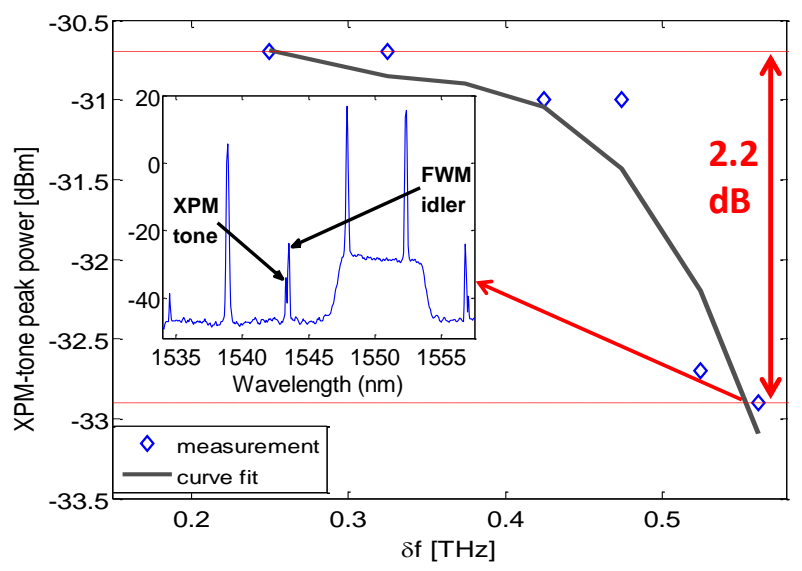

Fig. 2. Bandwidth measurement results for the photonic RFSA showing the XPM-tone peak power as a function of frequency detuning $\Delta f$. The inset is the recorded OSA spectrum for $\Delta f=562 \mathrm{GHz}$, showing that the generated XPMtone, and the FWM idler are about to overlap.

Figure 2 shows the bandwidth measurement results for the photonic RFSA showing the XPM-tone peak power as a function of frequency detuning $\Delta f$. For this measurement the output power of the EDFA 2 is set to $\sim 27 \mathrm{dBm}$. The minimum output power of the EDFA 2 for which the peak power of the generated XPM-tones will be still above the noise floor over the $3 \mathrm{~dB}$ bandwidth range of the photonic RFSA is $\sim 23 \mathrm{dBm}$. This results show that at a frequency detuning $\Delta f=562 \mathrm{GHz}$ the peak power reduction is $2.2 \mathrm{~dB}$ which is still within the 3 - 
$\mathrm{dB}$ bandwidth range of our all-optical RF spectrum analyzer. For the RFSA bandwidth measurement we were limited by the frequency range of the devices that we used, such as C-band limited laser sources and the available WDM coupler with a transmission band of only from $1547.5 \mathrm{~nm}$ to $1552.5 \mathrm{~nm}$. As we can see in the inset of the Fig. 2 , at $\Delta f=562 \mathrm{GHz}$ the generated XPM-tone which its peak power needs to be measured for the purpose of bandwidth measurement, is very close (almost overlapping) with the FWM idler generated from the interaction of the two TLSs. Further increasing the frequency detuning results in overlapping the XPM-tone that we measure with the FWM idler. Therefore, a minimum bandwidth of $560 \mathrm{GHz}$ is shown, whereas the actual bandwidth of our RF spectrum analyzer is $>560 \mathrm{GHz}$. Other factors that influence in limiting the bandwidth measurement are (1) the dispersion induced walk-off between the probe signal and the TLS signals, (2) the wavelength dependent propagation loss of the waveguide (see Fig. 3 (c) of [12]), which results in power drop variation at the wavelength range that is used for the bandwidth measurement. However, due to the equipment restriction the measurements were performed in this wavelength range; and (3) the polarization dependent loss that results in power drop variation over the wavelength range in which the bandwidth measurement is done. Optimization of the wavelength dependent propagation and polarization loss can be done by further investigations of the SRN waveguide's design.

We use our photonic RFSA to demonstrate monitoring of the RF spectrum of signals at rates of up to $\sim 160 \mathrm{GHz}$. The experimental setup of the all-optical RFSA is shown in Fig. 1. The signal under test is generated using a mode-locked laser (MLL) operating at $9.95 \mathrm{GHz}$, and $1550 \mathrm{~nm}$ with a pulse duration of $2 \mathrm{ps}$, and then passively multiplied by 4, 8, and 16 times (equivalent of $39.8 \mathrm{GHz}, 79.6 \mathrm{GHz}$, and $159.2 \mathrm{GHz}$, respectively) using an optical multiplexer (OMUX). The output of the OMUX passes through a polarization beam splitter in order to obtain a uniform polarization for all of the pulses at its output. This is due to the fact that the pulses which are generated using the OMUX have different polarizations from one pulse to another (in any period of $T=1 / f_{0}, f_{0}=9.95 \mathrm{GHz}$ ). The multiplexed signal is then amplified to $\sim 27 \mathrm{dBm}$. The probe signal is formed by generating a $\mathrm{CW}$ signal from a distributed feedback laser, which is then amplified to $\sim 18 \mathrm{dBm}$ followed by a $0.4 \mathrm{~nm}$ BPF to remove the out of band ASE noise. The signal under test and the $\mathrm{CW}$ probe are combined together using a WDM coupler with a transmission band of 5 $\mathrm{nm}$, centered at $1550 \mathrm{~nm}$. The output signal of the WDM coupler is then launched into the SRN waveguide. The output spectrum of the waveguide is measured using the OSA around the wavelength of the CW probe (XPM generated signal), which is the RF spectrum of the signal under test. We use two different OSAs with different resolutions in order to compare the results of the measurements: a high resolution OSA with a resolution as low as $20 \mathrm{MHz}$, and a conventional OSA with a resolution of $12.5 \mathrm{GHz}$. Figure 3 shows the recorded RF spectra of the signals at $\sim 10 \mathrm{GHz}, 40 \mathrm{GHz}, 80 \mathrm{GHz}$, and 160 $\mathrm{GHz}$, using the two mentioned OSAs. The insets show a zoomed version of the spectral lines.

Figure 3 (a) - (d) show the measured RF spectra using a conventional OSA with a $12.5 \mathrm{GHz}$ resolution for the pulse trains with repetition rates of $9.95 \mathrm{GHz}, 39.8 \mathrm{GHz}, 79.6 \mathrm{GHz}$, and $159.2 \mathrm{GHz}$, respectively. The information that can be received from the RF spectrum of a signal depends on the resolution of the OSA. As can be seen, the resolution is not high enough in Fig. 3 (a) to distinguish the spectral tones at multiples of $9.95 \mathrm{GHz}$, as the separation between the spectral tones is smaller than the resolution of the OSA. For the pulse trains shown in the Figs. 3 (b) - (d) the repetition rate is larger than the resolution of the OSA, making it possible to see some spectral content around the desired frequencies, but further details of the frequency content of the signal cannot be seen due to the coarse resolution of the OSA. Using a higher resolution OSA can provide further details of the RF spectrum of the signal. Figure 3 (e) shows the measured spectrum using the OSA with a $20 \mathrm{MHz}$ resolution for the pulse train with repetition rate of $9.95 \mathrm{GHz}$ (the OMUX is not used in this case). As can be seen the separation between the spectral lines is $9.95 \mathrm{GHz}$ as expected and equal to the repetition rate of the MLL. Figures 3 (f) - (h) show the measured spectra using the high resolution OSA for the pulse trains with
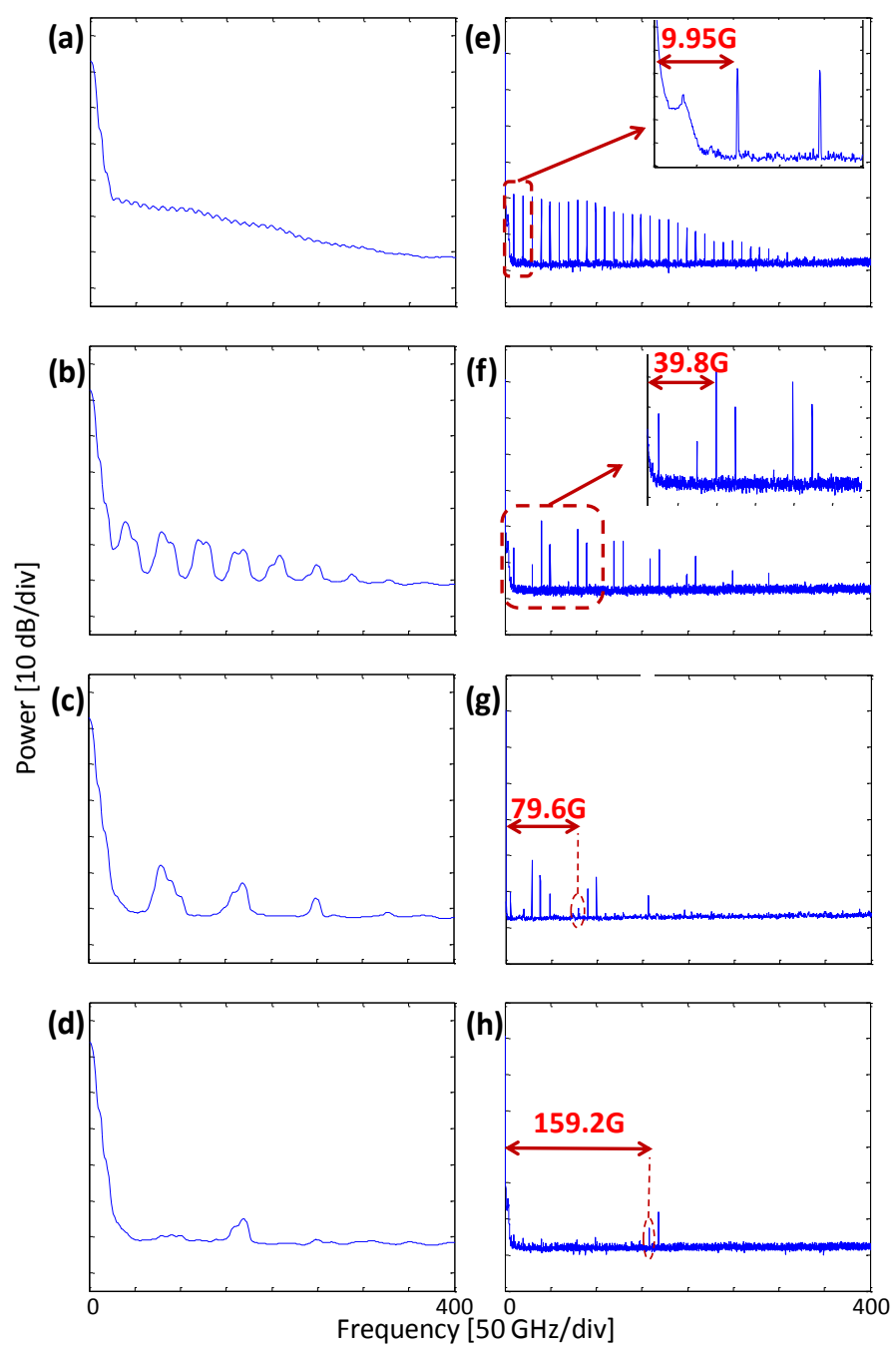

Fig. 3. Measured RF spectrum of pulse trains at (a) / (e) $9.95 \mathrm{GHz}$, (b) / (f) $39.8 \mathrm{GHz}$, (c) / (g) $79.6 \mathrm{GHz}$, and (d) / (h) $159.2 \mathrm{GHz}$ using a conventional OSA with $12.5 \mathrm{GHz}$ resolution / a high resolution OSA with $20 \mathrm{MHz}$ resolution. 
repetition rates of $39.8 \mathrm{GHz}, 79.6 \mathrm{GHz}$, and $159.2 \mathrm{GHz}$, respectively (the OMUX is used in these cases). The spectral tones representing the corresponding desired frequencies are highlighted in the figures, demonstrating the feasibility of alloptical RF spectrum analysis at these frequencies. Measurement of RF spectrum of pulse trains or data signals at higher bit rates (up to the operation bandwidth of the all-optical RFSA) are also possible, which require components capable of generation of signals at those frequencies. RFSA's results for the pulse trains in Figs. 3 (f) - (h) show the existence of spurious tones at some other frequencies in addition to the desired spectral tones of the corresponding pulse trains. This is due to the principal of operation of the OMUX. The OMUX passively multiplies the repetition rate of the intensity of its input data signal using an interferometric structure. It is designed to receive an PRBS optical data signal with a pattern length of $2^{7}-1$ at the bit rate of $9.95 \mathrm{GHz}$ and multiply the data rate by either 4,8 , or 16 at the output while preserving the pseudo randomness nature of the data signal, such that the output data signal will remain PRBS with a pattern length of $2^{7}-1$, regardless of its frequency. However, in our experiment we used a pulse train (instead of data signal) at repetition rate of $9.95 \mathrm{GHZ}$ as the input of the OMUX and therefore, the output is not a pure multiplied version of the input pulse train. This makes the corresponding spectrum of the output of the OMUX to include spurious tones at other frequencies, which will also be reflected to the recorded RF spectrum.

Comparing the spectra in Figs. 3 (a) - (d) with the corresponding spectra in Fig. 3 (e) - (h) we can see that the presence of the spurious tones is not explicitly clear in the OSA with a poorer resolution and therefore using an OSA with higher resolution will be advantageous. We need to mention that the resolution which can be achieved using the conventional electrical spectrum analyzers is in the order of tens of Hertz which provides a lot further details of the spectral content of the signal, whereas, using the all-optical RF spectrum analysis technique the best resolution of $\sim 1 \mathrm{GHz}$ is provided using the highest available resolution OSAs.

We see in the Fig. 3 that the spectral tones are vanished at frequencies $>\sim 300 \mathrm{GHz}$, whereas, the bandwidth measurement results in Fig. 2 demonstrate presence of spectral tones at frequencies > $560 \mathrm{GHz}$. This is due to the fact that the two tones in the bandwidth measurement experiment receive full amplification provided by the EDFA 2, but in the RFSA measurement of pulses the optical power provided by the EDFA 2 is distributed between all the spectral contents of the pulse source and the desired spectral tone (equal to the repetition rate of the output of the OMUX) does not receive full amplification. This can be resolved by either higher power allocation to the signal under test, or increasing the nonlinearity of the SRN platform to generate XPM peaks with higher power levels.

\section{CONCLUSIONS}

Performing integrated microwave photonic can reduce the overall footprint of the microwave photonic circuit. Siliconbased platforms such as SOI and silicon nitride have attracted lots of attention in the past decade, where many works have investigated microwave photonics application using silicon nitride platforms. The potential of reducing the insertion loss, broadband operation, and the absence of nonlinear loss make silicon nitride a suitable candidate for optical signal processing and microwave photonics applications. This work shows the potential of using SRN platforms for performing photonic RFSA.

In this paper we report the first demonstration of an XPMbased RFSA using an integrated silicon nitride waveguide. The measurements show a bandwidth of more than $560 \mathrm{GHz}$ for our photonic RFSA. Measurements of RF spectra for the pulse trains at repetition rates from $\sim 10 \mathrm{GHz}$ to $\sim 160 \mathrm{GHz}$ are demonstrated. By further optimization of the design of our SRN waveguide such as increasing the nonlinearity of the waveguide and more appropriate dispersion engineering, larger operation bandwidth for the photonic RFSA can be obtained.

\section{REFERENCES}

[1] C. Dorrer and D. N. Maywar, "RF spectrum analysis of optical signals using nonlinear optics," J. Lightwave Technol. 22(1), 266-274 (2004).

[2] M. Pelusi, F. Luan, T. D. Vo, M. R. E. Lamont, S. J. Madden, D. A. Bulla, D.-Y. Choi, B. Luther-Davies, and B. J. Eggleton, "Photonicchip-based radio frequency spectrum analyser with terahertz bandwidth," Nat. Photon. 3(3), 139-143 (2009).

[3] B. Corcoran, T. D. Vo, M. D. Pelusi, C. Monat, D X. Xu, A. Densmore, R. Ma, S. Janz, D. J. Moss, and B. J. Eggleton," Silicon nanowire based radio frequency spectrum analyzer," Opt. Express 18(19), 20190-20200 (2010).

[4] M. Ferrera, C. Reimer, A. Pasquazi, M. Peccianti, M. Clerici, L. Caspani, S. T. Chu, B. E. Little, R. Morandotti, and D. J. Moss, "Terahertz bandwidth integrated radio frequency spectrum analyzer via nonlinear optics," Opt. Express 22(18), 21488-21498 (2014).

[5] D. J. Moss, R. Morandotti, A. L. Gaeta, and M. Lipson, "New CMOScompatible platforms based on silicon nitride and Hydex for nonlinear optics," Nat. Photonics 7(8), 597-607 (2013).

[6] C. G. H. Roeloffzen, L. Zhuang, C. Taddei, A. Leinse, R. G. Heideman, P. W. L. Van Dijk, R. M. Oldenbeuving, D. A. I. Marpaung, M. Burla, and K.-J. Boller, "Silicon nitride microwave photonic circuits," Opt. Express 21(19), 22937-22961 (2013).

[7] B. J. Eggleton, B. Luther-Davies, and K. Richardson, "Chalcogenide photonics," Nat. Photonics 5(3), 141-148 (2011).

[8] J. Leuthold, C. Koos, andW. Freude, "Nonlinear silicon photonics," Nat. Photonics 4(8), 535-544 (2010).

[9] J. F. Bauters, M. J. R Heck, D. D. John, J. S. Barton, C. M. Bruinink, A Leinse, R. G. Heideman, D. J. Blumenthal, and J. E. Bowers, "Planar waveguides with less than $0.1 \mathrm{~dB} / \mathrm{m}$ propagation loss using wafer bonding," Opt. Express 19(24), 24090-24101 (2011).

[10] C. G. H. Roeloffzen, L. Zhuang, C. Taddei, A. Leinse, R. G. Heideman, P. W. L. van Dijk, R. M. Oldenbeuving, D. A. I. Marpaung, M. Burla, and K.-J. Boller, "Silicon nitride microwave photonic circuits," Opt. Express 21(19), 22937-22961 (2013)

[11] D. T. H. Tan, K. Ikeda, P. C. Sun, and Y. Fainman, "Group velocity dispersion and self phase modulation in silicon nitride waveguides,' Appl. Phys. Lett. 96, 061101 (2010).

[12] C. J. Krückel, A. Fulop, T. Klintberg, J. Bengtsson, P. A. Andrekson, and V. Torres-Company "Linear and nonlinear characterization of lowstress, high-confinement silicon-rich nitride waveguides," Opt. Express 23(20), 25827-25837 (2015).

[13] M. Rezagholipour Dizaji, C. J. Krückel, A. Fülöp, P. A. Andrekson, V. Torres-Company, and L. R. Chen, "Cross-phase-modulation-based wavelength conversion in low-stress silicon rich nitride waveguide," Conference on Optical Fiber Communications (OFC'16), 20-24 March 2016, Anaheim, CA.

[14] Xing Liu, Minhao Pu, Binbin Zhou, Clemens J. Krückel, Attila Fülöp, Victor Torres-Company, and Morten Bache, "Octave-spanning supercontinuum generation in a silicon-rich nitride waveguide," Opt. Lett.41, 2719-2722 (2016). 